EESTI NSV TEADUSTE AKADEEMIA TOIMETISED. IX KOIDE

FOOSIKALIS-MATEMAATILISTE JA TEHNILISTE TEADUSTE SEERIA. 1960, NR. 4

ИЗВЕСТИЯ АКАДЕМИИ НАУК ЭСТОНСКОИ ССР. ТОМ IX

СЕРИЯ ФИЗИКО-МАТЕМАТИЧЕСКИХ И ТЕХНИЧЕСКИХ НАУК. 1960, № 4

\title{
РЕШЕНИЕ НЕКОТОРЫХ ВАРИАНТОВ ЗАДАЧИ ПОЛЬКЕ
}

\section{А. ХУМАЛ,}

\author{
академик Академии наук Зстонской ССР
}

Одной из наиболее интересных проблем аксонометрии является перенесенная в центральную проекцию задача Польке: если в центральной проекции изображен прямоугольный равнобедренный тетраэдр, то как и по каким данным этого изображения оказывается возможным найти центр проекции и установить положение тетраэдра относительно картинной плоскости?

Довольно элементарное решение этой задачи недавно дал Н. В. Палувер*, причем выяснилось, что в соответствии с подбором данных имеется девять отличных друг от друга типов задачи. Для алгебраического решения этих девяти типов им получена система двух уравнений. Но возможны и такие частные случаи положения тетраэдра, которые не поддаются рассмотрению посредством этой системы, так как не все входящие в нее величины тогда существуют. Некоторые из таких частных случаев имеют наибольшее практическое значение. Как будет показано ниже, они также допускают элементарное решение задачи.

Наряду с частными случаями задачи Польке в центральной проекции здесь будет рассмотрено алгебраическое решение задачи в параллельной проекции, т. е. задачи Польке в ее первоначальном виде.

\section{1. Параметры задачи и исходные уравнения}

Пусть точки $O, A, B$ и $C$ означают (на фиг. 1) проекции вершин некоторого прямоугольного равнобедренного тетраэдра, а $P, Q$ и $R$ - точки схода его взаимно перпендикулярных ребер, общую вершину которых можно предполагать находящейся на картинной плоскости, следовательно, совпадающей с ее проекцией $O$. Длины отрезков $O A, O B, O C, O P, O Q$ и $O R$ и углы $A O B$ и $A O C$ полностью определяют имеющуюся плоскую фигуру; они и являются параметрами задачи и обозначены, соответственно, через $a, b, c, p, q, r, \alpha$ и $\beta$. Как известно, выходящие из центра S (находящегося в пространстве над черте-

* Н. В. П а лу в ер, Аналитический метод для построения аксонометрических систем координаг в центральной проекции, Труды Таллинского политехнического института. Серия A, № 120,1957 r.

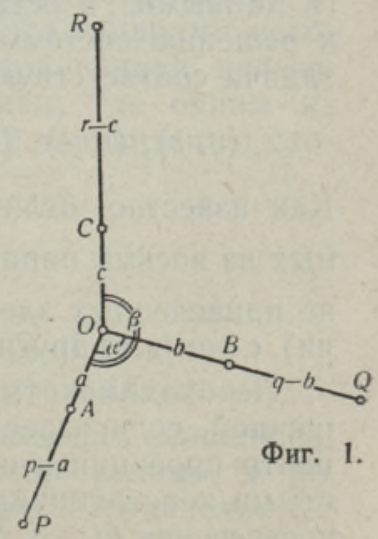


жом) лучи $\mathrm{S} P, \mathrm{~S} Q$ и $\mathrm{S} R$ взаимно перпендикулярны. На плоскости $\mathrm{S} O P$ (фиг. 2), проходящей через одно из ребер тетраэдра, легко установить,

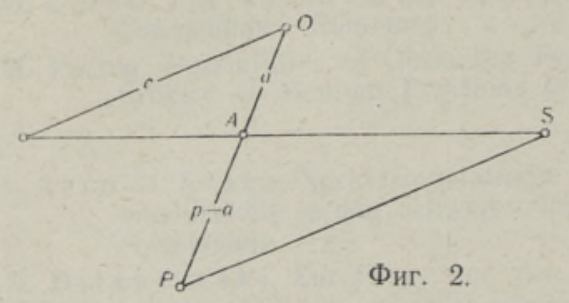
что длиной отрезка $\mathrm{S} P$ является $\frac{(p-a) e}{a}$, где $e$ означает длину ребра. Поскольку тетраэдр равнобедренный, то длинами отрезков $\mathrm{S} Q$ и $\mathrm{S} R$ являются, соответсгвенно, $\frac{(q-b) e}{b}$ и $\frac{(r-c) e}{c}$.

Квадрат длины отрезка $P Q$ можно получить как из прямоугольного треугольника $P S Q$, так и из косоугольного $P Q Q$, что приводит к уравнению .

$$
\left[\frac{(p-a) e}{a}\right]^{2}+\left[\frac{(q-b) e}{b}\right]^{2}=p^{2}+q^{2}-2 p q \cos \alpha .
$$

Таким же путем получаются (из треугольников $Q S R$ и $Q O R$ )

$$
\left[\frac{(q-b) e}{b}\right]^{2}+\left[\frac{(r-c) e}{c}\right]^{2}=q^{2}+r^{2}-2 q r \cos (\beta-\alpha)
$$

и (из треугольников RSP и ROP)

$$
\left[\frac{(r-c) e}{c}\right]^{2}+\left[\frac{(p-a) e}{a}\right]^{2}=r^{2}+p^{2}-2 r p \cos \beta .
$$

Эти равенства содержат, кроме параметров задачи, величину $e$ (длину взаимно перпендикулярных ребер тетраэдра). Ее легко устранить. Тогда остается система уравнений, содержащих только параметры задачи

$$
\left\{\begin{array}{l}
\frac{\left(\frac{p-a}{a}\right)^{2}+\left(\frac{q-b}{b}\right)^{2}}{p^{2}+q^{2}-2 p q \cos \alpha}=\frac{\left(\frac{r-c}{c}\right)^{2}+\left(\frac{p-a}{a}\right)^{2}}{r^{2}+p^{2}-2 r p \cos \beta} \\
\frac{\left(\frac{p-a}{a}\right)^{2}+\left(\frac{q-b}{b}\right)^{2}}{p^{2}+q^{2}-2 p q \cos \alpha}=\frac{\left(\frac{q-b}{b}\right)^{2}+\left(\frac{r-c}{c}\right)^{2}}{q^{2}+r^{2}-2 q r \cos (\beta-\alpha)} .
\end{array}\right.
$$

Поскольку восемь параметров задачи должны удовлетворять этим двум уравнениям, то шесть из них можно считать независимыми друг от друга данными, а оставшиеся два - искомыми. Итак, задача сводится к решению системы двух уравнений с двумя искомыми. Девять типов задачи соответствуют (по Н. В. Палуверу) следующим парам искомых:

$$
(q, r),(r, c),(b, r),(b, c),(\alpha, \beta),(c, \beta),(\beta, r),(b, \beta),(q, \beta) .
$$

Как известно, отличных друг от друга вариантов подбора двух искомых из восьми параметров имеется всего 28 (т. е. $\frac{8 \cdot 7}{2}$ ), но каждый из не приведенных здесь 19 вариантов совпадает (по ходу решения задачи) с тем или другим из указанных девяти типов.

Необходимо отметить, что задачу можно действительно считать решенной, если известен каждый из восьми параметров задачи, так как центр проекции и положение тетраэдра определяются ими весьма простыми конструкциями (ортогональной проекцией центра является точка пересечения высот треугольника $P Q R$, а расстоянием центра от картин- 
ной плоскости - геометрическое среднее расстояний той же точки пересечения от обонх концов высоты).

Для применения аксонометрических методов в центральной проекции исключительно важен случай, когда одно из трех взаимно перпендикулярных ребер тетраэдра находится на картинной плоскости (или параллельно ей) : ведь чаще всего картинная плоскость ставится вертикально, и одна из осей (т. е. одно ребро масштабного тетраэдра) обычно вертикальна. Но если одно из ребер тетраэдра находится на картинной плоскости и, следовательно, совпадает со своей проекцией, то это ребро не имеет точки схода. Например, если отрезок $O C$ является ребром тетраэдра, то нет точки $R$. В таком случае задача имеет только семь параметров и последние не могут удовлетворять полученной выше системе, поскольку оба уравнения содержат несуществующий параметр $r$.

Ясно, что теперь (фиг. 3) длинами отрезков $\mathrm{S} P$ и $\mathrm{S} Q$ являются по-прежнему $\frac{(p-a) e}{a}$ и $\frac{(q-b) e}{b}$, причем $e=c$; следовательно, остается в силе равенство

$\left(\frac{p-a}{a} c\right)^{2}+\left(\frac{q-b}{b} c\right)^{2}=p^{2}+q^{2}-2 p q \cos \alpha$.

Ввиду того, что прямая $O C$ перпендикулярна плоскости $P \mathrm{~S} Q$, она перпендикулярна и прямой $P Q$; это значит, что ортогональные проекции отрезков $O P$ и $O Q$ на прямой $O C$ равны друг другу:

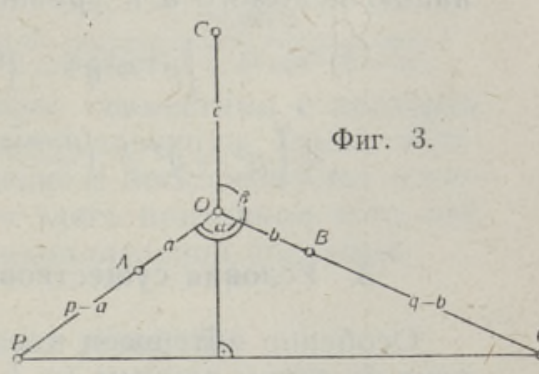

Этим уравнениям (I и II) должны удовлетворять параметры $a, b, c, p$, $q, \alpha$ и $\beta$ в случае, когда $O C$ - одно из трех взанмно перпендикулярных и равных по длине ребер тетраэдра. Тогда они и представляют собой исходные уравнения как при подсчете возможных вариантов задачи, так и при их решении.

\section{2. Варианты задачи}

Из семи параметров задачи, подчиненных двум уравнениям, пять могут быть данными и два - искомыми. Таким образом, имеется всего 21 (т. е. $\left.\frac{7 \cdot 6}{2}\right)$ вариант задачи. Но вследствие того, что второе уравнение содержит только четыре параметра ( $p, q, \alpha$ и $\beta)$, приходится исключить те варианты, в которых все эти четыре параметра одновременно принадлежат к данным, а именно три варианта с парами искомых $(a, b),(a, c)$ и $(b, c)$. Оставшиеся 18 вариантов задачи целесообразно разбить на 3 группы: во-первых, те варианты, где одним из искомых является $a, b$ или $c$; эта группа содержит 12 вариантов со следующими парами искомых:

$$
\begin{array}{cccc}
(a, p), & (a, q), & (a, \alpha), & (a, \beta), \\
(b, p), & (b, q), & (b, \alpha), & (b, \beta), \\
(c, p), & (c, q), & (c, \alpha), & (c, \beta) ;
\end{array}
$$

во-вторых, варианты с парами искомых $(p, \beta),(q, \beta)$ и $(\alpha, \beta)$; в-третьих, варианты с парами искомых $(p, q),(p, \alpha)$ и $(q, \alpha)$.

Решение вариантов задачи первой группы происходит одинаково; уравнение $p \cos \beta=q \cos (\beta-\alpha)$ содержит лишь одно искомое и решается довольно просто, затем легко решается и другое уравнение системы относительно оставшегося искомого. 
Аналогичен и ход решения задач второй группы: уравнение

$$
\left(\frac{p-a}{a} c\right)^{2}+\left(\frac{q-b}{b} c\right)^{2}=p^{2}+q^{2}-2 p q \cos \alpha
$$

не содержит $\beta$ и нетрудно решается относительно другого искомого, а затем $\beta$ легко вычисляется из уравнения $p \cos \beta=q \cos (\beta--\alpha)$.

Более интересно решение задачи в вариантах третьей группы. В случае пары искомых $(p, q)$ путем элиминации одного из них (например $p$ ) получается квадратное уравнение (относительно $q$ )

$$
\left(\frac{\cos ^{2}(\beta-a)}{a^{2} \cos ^{2} \beta}+\frac{1}{b^{2}}-\frac{\sin ^{2} \alpha}{c^{2} \cos ^{2} \beta}\right) q^{2}-2\left(\frac{\cos (\beta-a)}{a \cos \beta}+\frac{1}{b}\right) q+2=0 .
$$

Варианты же с парами искомых $(p, \alpha)$ и $(q, \alpha)$ приводят через элиминацию искомого $\alpha$ к уравнению четвертой степени

$$
\begin{gathered}
{\left[p^{2}+q^{2}-\left(\frac{p-a}{a} c\right)^{2}-\left(\frac{q-b}{b} c\right)^{2}\right]^{2} \sin ^{2} \beta+} \\
+\left[p^{2}-q^{2}+\left(\frac{p-a}{a} c\right)^{2}+\left(\frac{q-b}{b} c\right)^{2}\right]^{2} \cos ^{2} \beta=4 p^{2} q^{2} \sin ^{2} \beta .
\end{gathered}
$$

\section{3. Условия существования решений одного варианта задачи}

Особенно интересен вариант с искомыми $p$ и $q$, поскольку тогда задача по своим данным $(a, b, c, \alpha$ и $\beta)$ точно совпадает с задачей Польке в ее первоначальном смысле, т. е. когда рассматривается не центральная. а параллельная проекция. Как известно, решение задачи в параллельной проекции всегда существует (при единственном условии, что точки $O, A$; $B$ и $C$ не лежат на одной прямой). Поскольку параллельная проекция является предельным видом центральной проекции, то казалось бы возможным констатировать, что при данных $a, b, c, \alpha$ и $\beta$ всегда имеются решения задачи. Но соответствуюшее этим данным квадратное уравнение

$$
\left(\frac{\cos ^{2}(\beta-\alpha)}{a^{2} \cos ^{2} \beta}+\frac{1}{b^{2}}-\frac{\sin ^{2} \alpha}{c^{2} \cos ^{2} \beta}\right) q^{2}-2\left(\frac{\cos (\beta-a)}{a \cos \beta}+\frac{1}{b}\right) q+2=0
$$

не всегда имеет решения, а лишь при условии, что

$$
\left(\frac{\cos (\beta-a)}{a \cos \beta}+\frac{1}{b}\right)^{2} \geqslant 2\left(\frac{\cos ^{2}(\beta-\alpha)}{a^{2} \cos ^{2} \beta}+\frac{1}{b^{2}}-\frac{\sin ^{2} \alpha}{c^{2} \cos ^{2} \beta}\right) .
$$

Нетрудно видеть, что это неравенство сводится к более простому:

$$
\frac{a b \sin \alpha}{|a \cos \beta-b \cos (\beta-c)|} \geqslant \frac{c}{\sqrt{2}} \text {. }
$$

Естественно возникает вопрос, почему же уравнение, полученное для решения задачи, не всегда имеет решения, хотя и известно, что они сушеєтвуют. Дело же в том, что уравнение составлено для случая, когда одно из трех взаимно перпендикулярных ребер тетраэдра находится в картинной плоскости, причем составлено оно так, что искомым является $q$, т. е. расстояние точки схода $Q$ от вершины $O$. Таким образом, это уравнение имеет смысл только в типичных для центральной проекции условиях и не допускает тех положений тетраэдра, которые соответствовали бы решению задачи в параллельной проекции.

Следует еще отметить, что для решения задачи в параллельной проекции в случае, когда одно из трех взаимно перпендикулярных и равных 
по длине ребер тетраэдра находится в чертежной плоскости (например, ребро $O C$ ), требуется не пять данных, а только четыре, например, $a, b, \alpha$ и $\beta$. Действительно, если одно из ребер находится в чертежной плоскости, то оно перпендикулярно следу плоскости, проходящей через два остальных ребра; окружность, радиусами которой являются эти два ребра, пересекается со следом своей плоскости в точках $(E$ и $F$ на фиг. 4$)$, являющихся концами одного диаметра и вполне определенных данными $(a, b, \alpha \text { и } \beta)^{*}$.

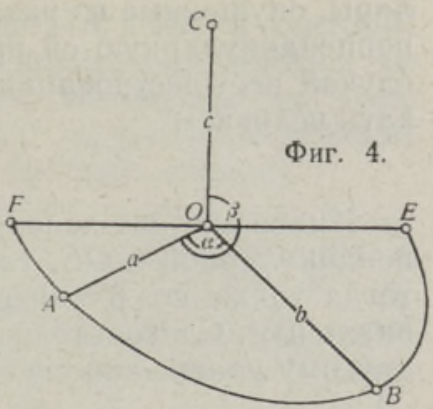

Интересно еще одно обстоятельство. Рассматриваемое квадратное уравнение превращается в линейное, если

$$
\frac{\cos ^{2}(\beta-a)}{a^{2} \cos ^{2} \beta}+\frac{1}{b^{2}}-\frac{\sin ^{2} \alpha}{c^{2} \cos ^{2} \beta}=0 \text {, т. е. если } c=\frac{a b \sin \alpha}{\sqrt{a^{2} \cos ^{2} \beta+b^{2} \cos ^{2}(\beta-a)}} .
$$

Это как раз то значение параметра $c$, которое совместимо с данными $a, b, \alpha$ и $\beta$ при решении задачи в параллельной проекции. Таким образом, пропажа (или, как говорится, превращение в бесконечность) одного решения квадратного уравнения является здесь признаком того, что задача с теми же данными разрешима и в параллельной проекции.

\section{4. Геометрические конструкции решений}

Пусть точки $O, A, B$ и $C$ означают проекции вершин некоторого прямоугольного равнобедренного тетраэдра (фиг. 5), причем одно из его трех взаимно перпендикулярных ребер находится в картинной плоскости и, следовательно, совпадает со своей проекцией $O C$. Данными задачи являются $a, b, c, \alpha$ и $\beta$; требуется найти центр проекции и положение тетраэдра.

Прежде всего можно констатировать, что в случае, когда точки $A, O$ и $B$ находятся на одной прямой, задача либо не имеет решений, либо имеет их бесконечное множество. Дело в том, что если прямые $O A$ и $O B$, являющиеся проекциями двух ребер тетраэдра, совпадают друг с другом, то центр проекции находится в проходящей через эти ребра плоскости,

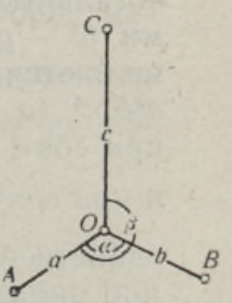

Фиг. 5. след которой проходит через точки $A, O$ и $B$ (являясь одновременно и прямой схода плоскости) и должен быть перпендикулярным к $O C$. Следовательно, задача не может иметь решений, если $A, O$ и $B$ находятся на прямой, которая не перпендикулярна к $O C$. Вместе с тем видно, что тогда уравнение $p \cos \beta=q \cos (\beta-\alpha)$, являвшееся исходным при решении задачи, не соответствует действительности (так как перпендику-

* Проекцией окружности является эллипс; даны его центр $O$ и пара сопряженных диаметров их конечными точками $A$ и $B$. Расстояние точки $E$ от центра можно вычислить по данным, например, следующим образом. Если центр эллипса совпадает с полюсом, диаметр длиною $2 a$ находится на полярной оси и сопряженный ему диаметр длиною $2 b$ отклоняется от полярной оси на угол $\alpha$, то уравненне эллипса в полярных координатах (气 и $\theta)$ будет $\varrho=\frac{a b \sin a}{\sqrt{a^{2} \sin ^{2} \vartheta+b^{2} \sin ^{2}(\vartheta-a)}}$ (что, впрочем, легко установить, исходя из уравнения того же эллипса в соответствующей системе декартовых иоординат $\left.\frac{(x-y \operatorname{ctg} a)^{2}}{a^{2}}+\frac{y^{2}}{b^{2} \sin ^{2} a}=1\right)$. Расстояние точки $E$ от центра выражается лросто как е при $\vartheta=\beta-\frac{\pi}{2} ;$ оно, следовательно, равно $a b \sin a$ $\sqrt{a^{2} \cos ^{2} \beta+b^{2} \cos ^{2}(\beta-a)}$ 
ляры, опущенные из различных точек $P$ и $Q$ одной прямой на другую, не перпендикулярную ей прямую, не могут совпасть друг с другом). Этот случай несуществования решений задачи можно коротко охарактеризовать условием

$$
\sin \alpha=0 \neq \cos (\beta-\alpha) \text {. }
$$

Иначе обстоит дело, когда точки $A, O$ и $B$ находятся на прямой, перпендикулярной к $O C$, т. е. когда $\sin \alpha=0=\cos (\beta-\alpha)$. Ввиду того, что тогда также $\cos \beta=0$, равенство $p \cos \beta=q \cos (\beta-\alpha)$ становится тривиальным. Следовательно, искомые $p$ и $q$ должны удовлетворять только другому уравнению системы

$$
\left(\frac{p-a}{a} c\right)^{2}+\left(\frac{q-b}{b} c\right)^{2}=p^{2}+q^{2}-2 p q \cos \alpha,
$$

в котором $\cos \alpha$ теперь равно либо - 1 , либо 1 , так что это уравнение сводится к следующему:

$$
\left(a^{2}-c^{2}\right) b^{2} p^{2} \pm 2 a^{2} b^{2} p q+a^{2}\left(b^{2}-c^{2}\right) q^{2}+2 a b^{2} c^{2} p+2 a^{2} b c^{2} q-2 a^{2} b^{2} c^{2}=0 .
$$

Как известно из теории кривых второго порядка, уравнение $g x^{2}+2 h x y+$ $+k y^{2}+2 l x+2 m y+n=0$ не имеет решений только в случаях $h^{2}-g k<0<(g+k)\left(g k n+2 h l m-g m^{2}-h^{2} n-k l^{2}\right)$ и $h^{2}-g k=0=$ $=g k n+2 h l m-g m^{2}-h^{2} n-k l^{2}>l^{2}+m^{2}-(g \perp k) n$, имеет единственное решение $\left(x=\frac{k l-h m}{h^{2}-g k}, y=\frac{g m-h l}{h^{2}-g k}\right)$ в случае $h^{2}-g k<0=g k n+$ $+2 h l m-g m^{2}-h^{2} n-k l^{2}$ и бесконечное множество решений во всех остальных случаях. Применительно кданному уравнению, где величина ми $h^{2}-g k, g+k, g k n+2 h l m-g m^{2}-\hbar^{2} n-k l^{2}$ и $l^{2}+m^{2}-(g+k) n$ являются, соответственно, $a^{2} b^{2} c^{2}\left(a^{2}+b^{2}-c^{2}\right), \quad 2 a^{2} b^{2}-\left(a^{2}+b^{2}\right) c^{2}$, $a^{4} b^{4} c^{4}(a \pm b)^{2}$ и $a^{2} b^{2} c^{2}\left(4 a^{2} b^{2}-a^{2} c^{2}-b^{2} c^{2}\right)$, это приводит к выводу, что при $\cos \alpha=-1$ уравнение всегда имеет бесконечное множество решений, а при $\cos \alpha=1$ имеет в случае $a^{2}+b^{2}-c^{2}<0=a-b\left(\right.$ т. е. $\left.a=b<\frac{c}{\sqrt{2}}\right)$ единственное решение $(p=a, q=a)$ и во всех иных случаях бесконечное множество решений. При этом предполагается, конечно, что точка $C$ не совпадает с точкой $O$ и что в случае совпадения точек $A$ и $B$ они не идентичны с точкой $O$, т. е. $\left(a^{2}+b^{2}\right) c \neq 0$.

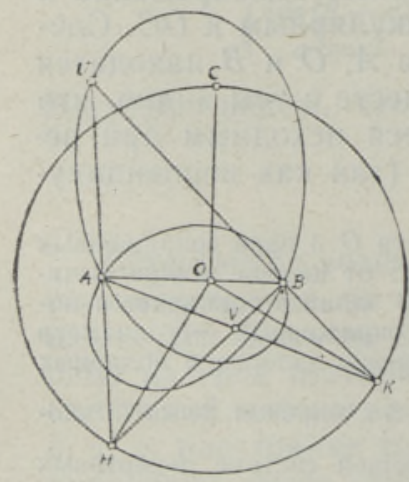

Фнг. 6.

Построение решений задачи в случае $\sin \alpha=$ $=0=\cos (\beta-\alpha)$ весьма просто (фиг. 6). Поскольку центр проекции находится на перпендикулярной к $O C$ плоскости, проходящей через прямую $A B$, и длина взаимно перпендикулярных ребер тетраэдра известна (она равна $c$ ), то известна в этой плоскости и та окружность, на которой лежат две вершины тетраэдра. Известно также, что установленные в них радиусы перпендикулярны друг другу. Но вместе с тем ясно, что каждая пара взаимно перпендикулярных радиусов представляет одно из возможных положений ребер тетраэдра. По каждой такой паре радиу сов легко найти соответствующий этому положению тетраэдра центр проекции. Например, если брать радиусы $O H$ и $O K$ (причем плоскость окружности повернута вокруг своего следа на картинную плоскость), то лучами, выходящими из 
центра проекции, могут быть либо $A H$ и $B K$, либо $A K$ и $B H$, а центром, соответственно, либо точка $U$, либо $V$. Из всех возможных центров на плоскости окружности образуется особая линия, состоящая из двух кривых четвертого порядка, симметричных друг другу относительно следа плоскости * (на фиг. 6 изображена одна из этих кривых).

Конструкция дает в случае совпадения точек $A$ и $B$ только такое решение, что центр проекции лежит на картинной плоскости в точке $A$ (идентичной с $B$ ). Хотя уравнение задачи при $\cos \alpha=1$ и $a=b=p=q$ явно удовлетворяется, это тривиальное решение означает вырождение проекции и поэтому, собственно, не может считаться решением задачи. Тривиальное решение оказывается единственным решением, если $a<\frac{c}{\sqrt{2}}$. Если же $a \geqslant \frac{c}{\sqrt{2}}$, то существует, кроме тривиального решения, бесконечное множество нетривиальных решений. Их конструкция сводится к построению таких хорд окружности, которые имеют длину $c \sqrt{2}$ и проходят через точку $A$ (или их продолжения проходят через $A$ ). При $a=\frac{c}{\sqrt{2}}$ имеется лишь одна такая хорда (фиг. 7), именно $H K$; следовательно, в этом

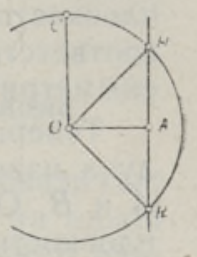

Фиг. 7. случае условиям задачи соответствует только одно положение тетраэдра (симметричное относительно картинной плоско-

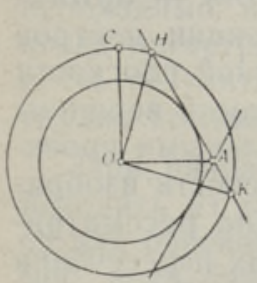

Фиг. 8. сти), но центром проекции может быть любая точка прямой $H K$ (за исключением, пожалуй, точек $A, H$ и $K$ ). При $a>\frac{c}{\sqrt{2}}$ для построения требуемых хорд цепесообразно использовать вспомогательную окружность с радиусом $\frac{c}{\sqrt{2}}$, так как эти хорды являются ее касательными. Как видно (фиг. 8), условиям за. дачи теперь соответствуют два положения тетраэдра, и возможные центры проекции составляют две прямые, проходящие через точку $A$.

Наконец, конструкция решений в общем случае, когда $\sin \alpha \neq 10$. Поскольку одно из трех взаимно перпендикулярных ребер тетраэдра находится в картинной плоскости, совпадая со своей проекцией, то остальные два из этих ребер находятся на плоскости, перпендикулярной к $О$ С. Следом плоскости является перпендикулярная к $O C$ прямая $g$, проходящая через $O$ (фиг. 9). В этой плоскости известна та

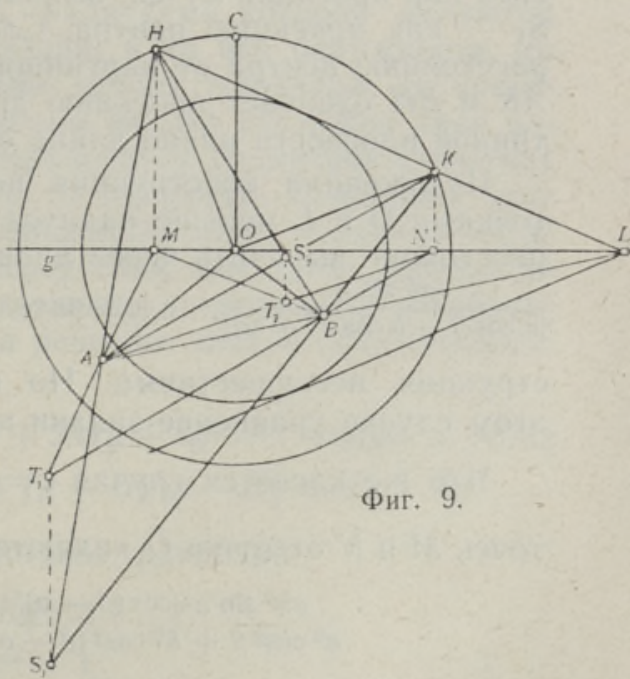

* Если за ось абсцисс взять след плоскости и за начало координат точку $O$, то уравнения кривых можно написать следующим образом:

$$
\begin{gathered}
c^{2}(x-a)^{2}(x+b)^{2}+\left[2 c^{2}(x-a)(x+b)-b^{2}(x-a)^{2}-a^{2}(x+b)^{2}\right] y^{2} \pm \\
\pm 2 a b(a+b) y^{3}+\left(c^{2}-a^{2}-b^{2}\right) y^{4}=0 .
\end{gathered}
$$


окружность, на которой находятся две вершины тетраэдра; известно также, что хорда между вершинами имеет длину $c \sqrt{2}$. Проекцией хорды является $A B$, а ее следом - точка пересечения прямых $A B$ и $g$ (обозначенная через $L$ ). Если повернуть плоскость окружности вокруг следа $g$ на картинную плоскость и начертить на ней также вспомогательную окружность с радиусом $\frac{c}{\sqrt{2}}$, то остается лишь построить касательные вспомогательной окружности, проходящие через точку L. (Ввиду того, что они симметричны друг другу относительно прямой $g$ и что плоскость окружности можно было повернуть на картинную плоскость либо в ту, либо в другую сторону, можно брать только одну из этих двух касательных.) Точки пересечения большей окружности и построенной касательной к меньшей, точки $H$ и $K$, являясь (повернутыми на картинную плоскость) вершинами тетраэдра, вполне определяют его положение, соответствующее данным задачи, - вернее, два положения тетраэдра, симметричных друг другу относительно картинной плоскости.

Теперь нетрудно найти центр проекции. Два выходящих из него луча известны: они проходят через вершины тетраэдра и их проекции $A$ и $B$. Ортогональные проекции этих лучей определены на картинной плоскости тем, что ортогональные проекции вершин тетраэдра находятся на прямой $g$ (они обозначены через $M$ и $N$ ), а точки $A$ и $B$ являются своими же ортогональными проекциями. Точка пересечения прямых $A M$ и $B N$ есть ортогональная проекция одного центра, соответствующего данным задачи, а точка пересечения прямых $A N$ и $B M$ - ортогональная проекция другого возможного центра; эти проекции центров обозначены через $T_{1}$ и $T_{2}$. Расстояния центров от картинной плоскости также легко установить: повернутые на картинную плоскость вершины тетраэдра, точки $H$ и $K$, могут считаться такими косоугольными проекциями этих вершин, что все расстояния от картинной плоскости изображаются в неискаженной величине; тогда $A H$ и $B K$ можно рассматривать как проекции лучей, выходящих из центра, а точку их пересечения $\mathrm{S}_{1}$ - как проекцию центра. Следовательно, длина отрезка $T_{1} \mathrm{~S}_{1}$ равна расстоянию центра от картинной плоскости. Точка пересечения прямых $A K$ и $B H$ означает проекцию другого центра, и его расстояние от картинной плоскости равно длине отрезка $T_{2} \mathrm{~S}_{2}$.

Изложенная конструкция неосуществима, если расстояние между точками $O$ и $L$ меньше радиуса вспомогательной окружности. Если это расстояние выразить через данные $a, b, \alpha$ и $\beta$, то его значением будет $\frac{a b \sin \alpha}{\mid a \cos \beta-b \cos (\beta-a)]}$, следовательно, при $\frac{a b \sin a}{|a \cos \beta-b \cos (\beta-a)|}<\frac{c}{\sqrt{2}}$ конструкция неосуществнма. Но как было показано выше, именно в этом случае уравнение задачи не имеет решений.

Что же касается случая $c=\frac{a b \sin a}{\sqrt{a^{2} \cos ^{2} \beta+b^{2} \cos ^{2}(\beta-c)}}$, то расстояниями точек $M$ и $N$ от точки $O$ являются тогда, соответственно,

$$
\frac{a b^{2} \sin \alpha \cdot|\cos (\beta-a)|}{a^{2} \cos ^{2} \beta+b^{2} \cos ^{2}(\beta-a)} \text { и } \frac{a^{2} b \sin \alpha \cdot|\cos \beta|}{a^{2} \cos ^{2} \beta+b^{2} \cos ^{2}(\beta-a)},
$$

вследствие чего прямая $A M$ параллельна прямой $B N$. В этом случае найденное положение тетраэдра допускает решение задачи в параллельной проекции, хотя и сохраняется другое решение в центральной проекции.

Конструкцию нельзя применять (она осуществима, но приводит к неверным результатам), если точка $A$ или точка $B$ находится на пря- 
мой $g$ (совпадая с $L$ ). Например, если $B$ совпадает с $L$, то $O B$ является ребром тетраэдра и не может иметь иную длину, как $c$; следовательно, задача теперь не имеет решений при $b \neq-c$. Ввиду того, что теперь нет точки схода $Q$, оба уравнения задачи недействительны; поэтому из них и не видно несуществования решений при $b \neq c$.

Вместе с тем ясно, что при $b-c=0=\cos (\beta-\alpha)$. ни один из существующих параметров задачи $(a, b, c, \alpha, \beta$ и $p)$ не может являться искомым. Действительно, если даны точки $O, B$ и $C$ так, что отрезки $O B$ и $O C$ перпендикулярны друг другу и равны по длине, то эти точки уже вполне определяют положение тетраэдра, но оставляют параметры $a, \alpha$ и $p$ совсем произвольными. Последние же все необходимы для определения центра проекции: его ортогональной проекцией на картинной плоскости служит точка схода $P$, а расстоянием от картинной плоскости является $\frac{(p-a) c}{a}$.

\section{5. Алгебраическое решение задачи в параллельной проекции}

Задачу Польке в ее первоначальном виде можно сформулировать так: на плоскости чертежа даны четыре точки, находящиеся не на одной прямой; считая их проекциями вершин некоторого прямоугольного равнобедренного тетраэдра, найти направление лучей проекции и положение тетраэдра относительно чертежной плоскости. Хотя ряд способов решения этой задачи посредством геометрических конструкций известен, необходимо иметь алгебраический способ решения для тех случаев, когда геометрические конструкции не могут обеспечить требуемую точность результатов ввиду того, что черчение связано с неизбежными и трудно контролируемыми неточностями.

При разработке алгебраического способа решения задачи можно исходить из тех же геометрических соображений, которые используются для обоснования какого-нибудь конструктивного решения ее. Например, если брать шаровую поверхность, радиусами которой являются взаимно перпендикулярные ребра тетраэдра, и на ней окружности, по которым она пересекается с плоскостями граней тетраэдра, то проекции этих окружностей определены данными весьма несложно (как эллипсы, для каждого из которых пара сопряженных диаметров известна) и дают возможность установить контур ее проекции. Поскольку этот контур состоит из следов тех лучей проекции, которые являются касательными шаровой поверхности, то посредством его легко устанавливается направление лучей, а затем и положение тетраэдра. В алгебраическом осуществлении намеченного хода решения задачи существенную роль играет следующая теорема:

кривые $G x^{2}+2 H x y+K y^{2}=1$ и $g x^{2}+2 h x y+k y^{2}=1$ имеют в своих обших точках общие касательные, если $(g-G)(k-K)=(h-H)^{2}$.

Доказывается теорема тем, что из системы уравнений

$$
\left\{\begin{array}{l}
G x^{2}+2 H x y+K y^{2}=1 \\
g x^{2}+2 h x y+k y^{2}=1
\end{array}\right.
$$

следует (если элиминировать $x$ )

$$
\left[(G k-g K)^{2}+4(G h-g H)(K h-k H)\right] y^{4}+
$$

$+2[(G k-g K)(g-G)-2(G h-g H)(h-H)] y^{2}+(g-G)^{2}=0$, 
откуда для существования кратного решения получается условие

$$
\begin{aligned}
& {\left[(G k-g K)^{2}+4(G h-g H)(K h-k H)\right](g-G)^{2}-} \\
& -[(G k-g K)(g-G)-2(G h-g H)(h-H)]^{2}=0,
\end{aligned}
$$

которое после некоторой перегруппировки члеков примет вид

$$
(G h-g H)^{2}\left[(g-G)(k-K)-(h-H)^{2}\right]=0,
$$

причем ясно, что если из системы уравнений элиминировать не $x$, а $y$, то получается, соответственно, условие

$$
(K h-k H)^{2}\left[(k-K)(g-G)-(h-H)^{2}\right]=0 ;
$$

оба же условия действительно выполнены * при

$$
(g-G)(k-K)=(h-H)^{2} .
$$

Теперь можно перейти к решению задачи Польке. Пусть даны точки $O, A, B$ и $C$, которые означают проекции вершин некоторого прямоугольного равнобедренного 'тетраэдра, причем общая вершина взаимно перпендикулярных ребер совпадает с $O$. Длины отрезков $O A, O B$ и $O C$ и величины углов $A O B$ и $A O C$ являются данными задачи и обозначены, соответственно, через $a, b, c, \alpha$ и $\beta$ (фиг. 5). Искомое направление лучей может быть представлено двумя искомыми величинами: пусть $\varphi$ означает угол между прямой $O A$ и проекцией нормали чертежной плоскости, а т - тангенс угла отклонения лучей проекции от нормали.

Шаровая поверхность с центром $O$, проходящая через остальные вершины тетраэдра, пересекается с проходящими через $O$ плоскостями граней теграэдра по окружностям, проекции которых являются эллипсами

$$
\begin{gathered}
\frac{x^{2}}{a^{2}}-\frac{2 \operatorname{ctg} a}{a^{2}} x y+\left(\frac{\operatorname{ctg}^{2} a}{a^{2}}+\frac{1}{b^{2} \sin ^{2} c}\right) y^{2}=1, \\
\frac{x^{2}}{a^{2}}-\frac{2 \operatorname{ctg} \beta}{a^{2}} x y+\left(\frac{\operatorname{ctg}^{2} \beta}{a^{2}}+\frac{1}{c^{2} \sin ^{2} \beta}\right) y^{2}=1 \\
\left(b^{2} \sin ^{2} \alpha+c^{2} \sin ^{2} \beta\right) x^{2}-2\left(b^{2} \sin \alpha \cos \alpha+c^{2} \sin \beta \cos \beta\right) x y+ \\
+\left(b^{2} \cos ^{2} \alpha+c^{2} \cos ^{2} \beta\right) y^{2}=b^{2} c^{2} \sin ^{2}(\beta-\alpha),
\end{gathered}
$$

если прямая $O A$ считается осью абсцисс и точка $O$ началом координат**. Контуром проекции шара будет огибающий их эллипс, имеющий тот же центр $O$. Следовательно, в его уравнении $g x^{2}+2 h x y+k y^{2}=1$ коэффициенты $g, h$ и $k$ можно найти с помощью приведенной теоремы. Действительно, при обозначениях

* Они также выполнены при $G h-g H=0=K h-k H$, но легко убедиться в том, что тогда кривые либо совпадают (если $g=G$ ), либо не имеют ни одной общей точки (если $g \neq G$ ).

** Уравнение первого эллипса $\frac{(x-y \operatorname{ctg} a)^{2}}{a^{2}}+\frac{y^{2}}{b^{2} \sin ^{2} \alpha}=1$ перепишется в полярных координатах так: $\varrho^{2}\left[a^{2} \sin ^{2} \theta+b^{2} \sin ^{2}(\vartheta-\alpha)\right]=a^{2} b^{2} \sin ^{2} a ;$ следовательно, уравнение третьего эллипса будет $\mathrm{Q}^{2}\left[b^{2} \sin ^{2}(\theta-\alpha)+c^{2} \sin ^{2}(\theta-\beta)\right]=b^{2} c^{2} \sin ^{2}(\beta-\alpha)$, что выше и переписано в декартовых координатах. 


$$
\begin{gathered}
\frac{1}{a^{2}}=G, \quad-\frac{\operatorname{ctg} a}{a^{2}}=H_{1}, \quad-\frac{\operatorname{ctg} \beta}{a^{2}}=H_{2}, \\
\frac{\operatorname{ctg}^{2} a}{a^{2}}+\frac{1}{b^{2} \sin ^{2} \alpha}=K_{1}, \quad \frac{\operatorname{ctg}^{2} \beta}{a^{2}}+\frac{1}{c^{2} \sin ^{2} \beta}=K_{2},
\end{gathered}
$$

$\frac{b^{2} \sin ^{2} \alpha+c^{2} \sin ^{2} \beta}{b^{2} c^{2} \sin ^{2}(\beta-\alpha)}=G_{3},-\frac{b^{2} \sin \alpha \cos \alpha+c^{2} \sin \beta \cos \beta}{b^{2} c^{2} \sin ^{2}(\beta-\alpha)}=H_{3}, \frac{b^{2} \cos ^{2} \alpha+c^{2} \cos ^{2} \beta}{b^{2} c^{2} \sin ^{2}(\beta-\alpha)}=K_{3}$

они определяются условием

$$
\left\{\begin{array}{l}
(g-G)\left(k-K_{1}\right)=\left(h-H_{1}\right)^{2} \\
(g-G)\left(k-K_{2}\right)=\left(h-H_{2}\right)^{2} \\
\left(g-G_{3}\right)\left(k-K_{3}\right)=\left(h-H_{3}\right)^{2}
\end{array}\right.
$$

или равносильной ему системой

$$
\left\{\begin{array}{l}
(g-G)\left(k-K_{1}\right)=\left(h-H_{1}\right)^{2} \\
\left(K_{2}-K_{1}\right)(g-G)-2\left(H_{2}-H_{1}\right)\left(h-H_{1}\right)+\left(H_{2}-H_{1}\right)^{2}=0 \\
\left(K_{3}-K_{1}\right)(g-G)-2\left(H_{3}-H_{1}\right)\left(h-H_{1}\right)+\left(G_{3}-G\right)\left(k-K_{1}\right)- \\
-\left(G_{3}-G\right)\left(K_{3}-K_{1}\right)+\left(H_{3}-H_{1}\right)^{2}=0 .
\end{array}\right.
$$

Для краткости можно ввести еще следующие обозначения:

$$
\begin{gathered}
t=g-G, \gamma=\frac{K_{2}-K_{1}}{2\left(H_{2}-H_{1}\right)}, \quad \delta=\frac{H_{2}-H_{1}}{2}, \quad \lambda=2 \gamma \cdot \frac{H_{3}-H_{1}}{G_{3}-G}-\frac{K_{3}-K_{1}}{G_{3}-G}, \\
\mu=\frac{H_{3}-H_{1}}{G_{3}-G}\left(H_{2}-H_{3}\right)+K_{3}-K_{1} .
\end{gathered}
$$

Тогда из второго уравнения системы следует $h-H_{1}=\gamma t+\delta$, а из третьего $k-K_{1}=\lambda t+\mu$, так что первое уравнение будет

$$
t(\lambda t+\mu)=(\gamma t+\delta)^{2} .
$$

Следовательно, $t$ вычисляется как решение уравнения

$$
\left(\lambda-\gamma^{2}\right) t^{2}+(\mu-2 \gamma \delta) t-\delta^{2}=0 .
$$

Это уравнение имеет одно отрицательное решение и одно положительное, и из них используется только отрицательное решение, т. е.

$$
t=\frac{\left.2 \gamma \delta-\mu-\sqrt{\mu^{2}+4 \delta(\delta \lambda-\gamma \mu}\right)}{2\left(\lambda-\gamma^{2}\right)} .
$$

Дело в том, что данные три эллипса можно огибать одним эллипсом снаружи и другим изнутри; для определения обоих получена одна и та же система уравнений. Но первый из них больше и второй меньше, чем данные эллипсы. Поскольку в случае эллипса в уравнении $g x^{2}+2 h x y+$ $+k y^{2}=1$ коэффициенты $g$ и $k$ положительны и $g+k$ при увеличении эллипса уменьшается, а из уравнения $(g-G)\left(k-K_{1}\right)=\left(h-H_{1}\right)^{2}$ видно, что искомые $g$ и $k$ отклоняются от $G$ и $K$ в одну и ту же сторону, то $g-G$ действительно должно быть отрицательным.

После того как найдено $t$ и вычислены $g, h$ и $k$ (как известно, $g=t+G, h=\gamma t+\delta+H_{1}$ и $\left.k=\lambda t+\mu+K_{1}\right)$, целесообразно вычислить 
еще $\sqrt{(k-g)^{2}+4 h^{2}}$, обозначаемое в дальнейшем через $v$. Для вычисления искомых $\varphi$ и $\tau$ получаются следующие формулы *:

$$
\operatorname{tg} \varphi=\frac{k-g-v}{2 h}, \quad \tau=\sqrt{\frac{2 v}{g+k-v}},
$$

а радиус шара (т. е. длина взаимно перпендикулярных ребер тетраэдра) будет $\sqrt{\frac{2}{g+k+v}}$.

Наконец, что касается положения тетраэдра, то его вершины могут быть найдены как точки пересечения шаровой поверхности

$$
x^{2}+y^{2}+z^{2}=\frac{2}{g+k+v}
$$

с проходящими через точки $A, B$ и $C$ лучами

$$
\begin{gathered}
\frac{x-a}{\tau \cos \varphi}=\frac{y}{\tau \sin \varphi}=-z, \quad \frac{x-b \cos a}{\tau \cos \varphi}=\frac{y-b \sin a}{\tau \sin \varphi}=-z \\
\text { и } \frac{x-c \cos \beta}{\tau \cos \varphi}=\frac{y-c \sin \beta}{\tau \sin \varphi}=-z .
\end{gathered}
$$

Поступила в редакцию 11. X 1960

\title{
POHLKE ULESANDE MÕNE VARIANDI LAHENDAMINE
}

\section{A. Humal,}

\author{
Eesti NSV Teaduste Akadeemia akadeemik
}

\section{Resümee}

K. W. Pohlke ülesande, ülekantuna tsentraalprojektsiooni, on algebraliselt lahendanud hiljuti N. Paluver üllatavalt elementaarsel viisil. Kôrvale jäi aga erijuhtum, kui üks telgedest on pildipinnal.

Selgub, et ülesanne on sel juhul samuti elementaarselt lahendatav. Peavariandi jaoks tuuakse ka lahendi geomeetriline konstruktsioon.

Pohlke ülesannet tema algkujul, nagu teda tuntakse paralleelprojektsioonis juba 1860. aastast, võimaldab kaunis kergesti algebraliselt lahendada järgmine teoreem: kõverjoontel $G x^{2}+2 H x y+K y^{2}=1$ ja $g x^{2}+2 h x y+k y^{2}=1$ on ühistes punktides ühised puutujad, kui $(g-G)(k-K)=(h-H)^{2}$.

\section{Saabus toimetusse}

$$
\text { 11. X } 1960
$$

* Если уравнение $g x^{2}+2 h x y+k y^{2}=1$ переписать в полярных координатах, то получается $Q^{2}\left(g \cos ^{2} \theta+2 h \cos \theta \sin \theta+k \sin ^{2} \theta\right)=1$. Отсюда видно, что максимальное e соответствует такому $\theta$, при котором $g \cos ^{2} \theta+2 h \cos \vartheta \sin \theta+k^{2} \sin ^{2} \theta$ принимает наименьшее значение. Нетрудно установить, что подходящее $\theta$ получается из условия $\operatorname{tg} \vartheta=\frac{k-g-V\left(\overline{k-g)^{2}+4 \overline{h^{2}}}\right.}{2 h}$. Такое $\vartheta$ и обозначено через $\varphi$.

Поскольку рассматриваемый эллипс является контуром параллельной проекции шара, то его меньшая полуось равна радиусу шара, а отношение меньшей и большей полуосей равно косинусу угла отклонения лучей проекции от нормали чертежной плоскости. Из уравнения эллипса следует, что $Q^{2}$ имеет минимальное значение $\frac{2}{g+k+v}$ и максимальное $\frac{2}{g+k-v}$; их отношение $\frac{g+k-v}{g+k+v}$ будет, следовательно, $\frac{1}{1+\tau^{2}}$. 
DIE LOSUNG EINIGER VARIANTEN DER POHLKESCHEN AUFGABE

A. Humal,

Mitglied der Akademie der Wissenschaften der Estnischen SSR

\section{Zusammenfassung}

Die Aufgabe von K. W. Pohlke, übertragen in die Perspektive, wurde kürzlich vor N. Paluver algebraisch in überraschend elementarer Weise gelöst. Beiseite blieb jedoch der Sonderfall, dass eine der Achsen auf der Bildebene liegt.

Es stellt sich nun heraus, dass die Aufgabe in diesem Falle ebenso elementar lösbar ist. Für die Hauptvariante wird auch eine geometrische Konstruktion der Lösung gebracht.

Die Pohlkesche Aufgabe in ihrer ursprünglichen Form (wie sie in der Parallelprojektion schon seit 1860 bekannt ist) wird algebraisch ziemlich leicht lösbar unter Verwendung des Satzes: die Kurven $G x^{2}+2 H x y+K y^{2}=1$ und $g x^{2}+2 h x y+k y^{2}=1$ haben in ihren gemeinsamen Punkten gemeinsame Tangenten, wenn $(g-G)(k-K)=(h-H)^{2}$. 